\title{
TRADING OLD FOR NEW: ECONOBIOLOGY AND ECONOPHYSICS AS EXPLANATORY FRAMEWORKS OF CURRENT FINANCIAL SYSTEM
}

\author{
Iuliana Maria Ursu ${ }^{1} \&$ Raluca Simina Bilti ${ }^{2}$
}

DOI 10.1515/JHEEC-2016-0004

\begin{abstract}
The present study aim is to deliver a succinct overview of the existing literature concerning economic systems, and in particular financial systemsfrom the Econobiology, or the "evolutionary economics" perspective, mainly treated within the Adaptive Market Hypothesis, and the Econophysics perspective. In the heterodox frame, both the A.M.H. and the Econophysics are trying to explain the complexity of financial markets from $a$ "bottom up" perspective, hence "macroscopic" properties are viewed as the result of interactions at the level of the 'microscopic' constituents (Rickles, 2011, p.531-565). Given the expanded level of information we can access nowadays, we consider that an important attention should be given to the inclusion of both perspectives as explanatory frameworks of the financial markets.
\end{abstract}

JEL Classification: $\quad$ D53, G14, B26, B59, B50, D47

\section{Introduction}

In the post-modern times we are living in, with interconnected financial markets, and an implicit high level of sensibility in existing motions, one of the most important problems that require our attention pertains to the way capital market mechanisms function. Even

\footnotetext{
${ }^{1}$ West University of Timisoara, Author, Iuliana Maria Ursu - iuliana.m.ursu@gmail.com

${ }^{2}$ West University of Timisoara, Author,Raluca Simina Bilti - raluca_simina92@yahoo.com
} 
though an efficient market is an ideal environment for those participating in it, a series of arguments can be made against the Efficient Market Hypothesis, and through the progress in researching financial markets it has been proven that these tenants are not infallible, and models of transaction based on this theory do often times generate faulty results.

Beyond the implicit negative effects of financial turbulences, those can also open possible further avenues of research, or the opportunity of assessing the validity of the already classic ones under the light of new empirical evidences.

Defined by the random walk hypothesis, the standard model of finance it can be elusively summarized within a few simple hypotheses: (1) Gaussian distribution of prices; (2) current price is the best estimate of future price; (3) rational expectations; (4) equilibrium between offer and demand.

The principles were then used as a ground for the theoretical framework of the Efficient Market Hypothesis, which was to become the foundation of modern finances. The theory, postulated by Eugene Fama in 1965, and swiftly summarized in the epigram "asset prices fully reflect all available information"(Fama 1991, p. 1575), sparked controversy within economic researchers, as its conceptual shell relies on a series of „volatile” hypothesis, such as: (1) the absence of arbitrage opportunities; (2) rational investors, respectively rational price behaviour; (3) optimal processes of rational agents with respect to a given utility function.

The need of explaining economic phenomena in terms of micro-structures (i.e. agents) behaviours, while relying on the safety of "stylized facts”, indicates to some degree a biased approach.

The emergence of behavioural finance, and later on of different alternative theories, appeared as response to the need of a broader understanding of complex economic phenomena. The present study aim is to deliver a sketchy perspective over the existing literature regarding two of the most interesting approaches when it comes to understanding financial markets mechanisms: the Econobiology, or the "evolutionary economics" perspective, mainly treated within the Adaptive Market Hypothesis, and the Econophysics perspective.

\section{The standard (neo-classical) model of finance}

Defined by the random walk hypothesis, the standard model of finance it can be elusively summarized within a few simple hypotheses: (1) Gaussian distribution of prices; (2) current price is the best estimate of future price; (3) rational expectations; (4) equilibrim between offer and demand.

Based on those principles emerged the Efficient Market Hypothesis, defined within "asset prices fully reflect all available information"(Fama 1991, p. 1575). Like any other theory, EMH has sparked controversy in the economic world. One of those is represented by the idea of "instant" incorporation of all available information within the intrinsic value of the action (Fama 1965, pp. 34-105). Practically, because of the uncertainty that the new information is triggering, their „instant incorporation" has two implications: firstly, prices will over adjust initial changes to the intrinsic value, as often 
as they will under adjust. Secondly, the difference between the full adjustment of new prices to new successive values of intrinsic value, will in itself be independent, a random variable, with market price adjustments sometimes taking place before an event expected by the market, sometimes after the triggering of the event.

Another controversy is the trinomial Rationality $\rightarrow$ Random Movement $\rightarrow$ Information Efficiency. First, the informational efficiency implies the existence of a constant equilibrium between the price of the financial assets and the informational ensemble, their variations (i.e. prices) being due only to the arrival of new information on the market, arbitrary by definition, and which implicitly would determine a random behaviour of prices, respectively the impossibility of arbitration. As the price movement sequences are more random, the more efficient the market is and the imperfections of information gathering would lead to a decrease in information efficiency.

In turn, the random movement is conditioned by the existence of investment rationality, a concept that triggered many debates over time, contributing to the creation of an economic scene divided between the supporters of the rationality hypothesis, and implicitly the informational efficiency, on the one hand, and economists who reject the rationality hypothesis, and the martingale in describing price movements, respectively rejecting informational efficiency.

The Efficient Market Hypothesis limits are found within the limits of its abstractions (rationality, constant distribution of income and the relationship between risk and profitability, etc.), and within the incapacity of faithfully assessing the impact of exogenous factors on prices mechanisms. However, the theory keeps its place on the podium, as there is no accepted "correct" model of evaluating the intrinsic values of stocks.

The proximity of 1990 marks an amplification of disputes with the emergence of empirical studies by psychologists Daniel Kahneman and Amos Nathan Tversky, and the development of behavioural finance. The significant impact of this new field lies in its focus, namely the desire to understand the structure of the decision-making processes of investors, and more than that, to explain the dynamics of financial markets as a result of the behaviours of the groups of actors acting on the market.

The most common cognitive heuristics that attempt to highlight the irrational behaviour of investors are: heuristics of representativeness, anchoring, flocking, excess confidence. Besides these heuristics, another impact on how investors build their decision-making system is represented by to a series of forgeries, such as: aversion to loss, mental accounting, aversion to regret, etc.

Except behavioural biases, deviations from efficiency are also the market anomalies, which we find in three main forms: fundamental anomalies, technical anomalies and temporal anomalies.

Beyond the limits of the theory, E.M.H. has been accepted over time, mainly due to the stability of financial markets over a time horizon of more than 20 years (the period following the Great Depression - 1930, until 2000).

However, as it can be observed in the last decade, capital markets are characterized by ever-growing instability and volatility, suggesting the idea of different financial mechanisms, with faster, bigger and more diverse markets, than at any other point in modern history. The rising trend of anomalies in the last years addresses a series of 


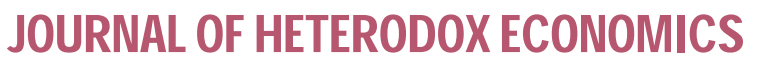

questions for economists concerning the possible existence of a more precise model for explaining the behaviours within the capital market.

\section{Alternatives models of finance}

When speaking about financial markets the topic of complex systems is not one to be ignored. We can define those systems in terms of some of their characteristics, namely (but not only): (1) a structure containing multiple substructures (units); (2) the existence of interdependence amongst those units; (3) a nonlinear interaction between the units; (4) a change in the system appears as the result of changes inside system's subunits.

If assuming a complex-adaptive system, we assume the hypothesis that system subunits adapt their behaviour with respect to the ever changing environment, this process thus generating a new structure in terms of its defining properties. Moreover, is the system is self-organizing adaptive, then as a result of the changing environment, the subunits modifies their own properties and behaviours, that at their turn affects the defining properties of the macrostructure.

Being characterized by the prior mentioned mosaic of properties, and also a series of „attributes” such as - fat tails, volatility clustering, persistence (long memory), multifractality, autocorrelation, or the existence of extreme events, the financial system is an obvious candidate for the complex systems typology. However, the question that remains is about the category in which to be framed: is it adaptive? Is it self-organizing adaptive?

Two of the main perspectives regarding the way market mechanisms are functioning are represented by the Econobiology, or the "evolutionary economics" mainly treated within the Adaptive Market Hypothesis, and the Econophysics perspective.

\subsection{The Adaptive Market Hypothesis (A.M.H.)}

A.M.H. is based on the meta-hypothesis that the functioning of the financial markets is carried out under conditions of non-uniform informational efficiency, which changes along their evolutionary trajectory. These changes occur both under the impact of structural, functional and institutional factors, as well as due to factors of a psychological and socio-behavioural nature.

The ultimate purpose of the theory is to identify the way in which human behavior responds to dynamic market conditions and to model the changes in behavior as a function of the environment in which the investors are engaging, having the following major implications:

Within the paradigm, the trade-off between risk and return is not stable in time and circumstances, varying according to the behavioral typologies of market agents and the trading environment.

Contrary to E.M.H., this hypothesis accepts the existence of arbitrage as from an evolutionary point of view "opportunities for profit are inherent to markets 
characterized by a high degree of liquidity". The level of market efficiency is directly correlated with the level of adaptability of the participants to the market environment. The success or failure of investment strategies depends on market conditions. In order to achieve a diversification within interconnected markets driven by macroeconomic factors, portfolio assets allocation should be adapted to the current environment, thus a higher diversity is required.

Innovation is the key to survival. The fourth implication of the A.M.H. has to do with the possibility that alphas' have values that differ from zero in certain time spans, a variation brought upon by the technological impact, innovation and competition inherent in the market.

Asset allocation is under a different perspective under A.M.H..Given the added importance of risk-return ratio for investors, A.M.H. suggests an allocation in terms of units of risk, rather than numerical values of portfolio weights. This type of strategy can be more practical and stable from a decision making perspective.

The main implication of assuming a non-uniform evolutionary pattern in terms of informational efficiency on market pricing mechanisms is that they deviate from "no memory" processes, hence the return movement does not follow a Brownian, Martingale or semi-Martingale movement. Furthermore, we can also encounter not normal distributions, fat tails, short- and long-term correlations, multifractality and persistent or anti-persistent behavior (characterized by shifts in the growth direction more frequent that a random process).

As attested by previous studies, some of the potential variables that can generate periods of deviation from market efficiency are represented by psychological biases, market microstructure particularities, the existence of market imperfections, nonoptimal investment behavior, etc. In this context, A.M.H. sees the efficient market hypothesis as an unattainable economical utopia, but well-equipped to be used as a reference point in measuring market efficiency.

Even though A.M.H. is of an abstract and qualitative nature, the hypothesis comes with some important practical applications for portfolio management. In this sense, one of the most significant applications relates to the risk premium, derived from the relationship between risk and return, a relationship defined by the market size and the market players' preferences. According to A.M.H., these preferences vary over time, thus influencing risk premium profile. The main implication is the existence of cycles of predictable and non-predictable patterns in stock prices (Lo, 2004) due to a constant investor flux - some chose to continue, while others exit the market with their numbers being constantly replenished by new entries.

The same principles can be applied in explaining the existence of temporary arbitrage opportunities: market players' entries and exits generate states of complex dynamics within the financial markets, where arbitrage opportunities appear and are exploited before completely vanishing. (Soteriou, Svensson, 2017, p. 12)

The limitations of the theory pertain to the existence of complementary variables that can impact the allocation strategies according to risk, such as returns variation, the existing correlation between different categories of assets, etc., conditions that presuppose the existence of a wider spectrum approach as far as control is concerned, probably through the development and implementation of systems that take into 
consideration all parameters relevant to the context of the investment: political or administrative specificity, estimation errors, cultural factors, etc.

Consequently, the testing of the A.M.H. must employ a multi-faceted approach, including, but not limited to: an analysis of the fundamental statistical properties of data in terms of heteroscedasticity, distribution parameters, linear and nonlinear auto-correlation, the existence of structural breaking points or the highlighting of fat tail-type effects.

Due to technological developments, market regulations, number of investors, their investment profile variety, and so on, market efficiency is expected to vary in cyclical patterns (Kim, Shamsuddin, \& Lim, 2011, p. 868-879). To this extent the empirical testing of adaptive market hypothesis is usually done based on linear and nonlinear dependence tests. The linear tests have been widely used in literature in order to test for autocorrelation in market returns, as for example in the case of random walk theory (Hoque, Kim, \& Pyun, 2007, p. 488-502). However, as observed by Butler and Kazakov, in their study "Testing implications of the Adaptive Market Hypothesis via computational intelligence", these tests should be complemented by a nonlinear test, for the purpose of targeting the cyclicality of predictability of market returns (Butler \& Kazakov, 2012, p.249-256).

\section{Tests for linear independence in the context of A.M.H.}

One of the post popular linear tests is represented by the Automatic Variance Ratio (VR) test, developed by Lo and MacKinlay in 1988, and applicable for every difference in holding periods. However, when testing the stock market predictability on several holding periods VR test can be inconvenient.

In this context, Chow and Denning developed a joint test based on the standard variance ratio test. The main improvement of the new one lies in the inclusion of multiple holding periods, while the pitfall arise due to the fact that only the maximum autocorrelation is taken into account, and that returns are assumed to be independently and identically distributed (the conditional heteroscedasticity in small samples is ignored). As Kim (2006) observed, the assumption of independent and identically distributed variables(i.d.d.) could lead to underestimation of uncertainty levels of the estimates (Kim 2006, p.38-43).

Further along, in order to overcome this impediment, Kim (2006) proposed an alternative based on the VR by Lo and MacKinlay (1988) and the joint test by Chow and Denning (1993), used to approximate the distribution of variance in the market returns and improve the small sample properties of variance ratio tests (Soteriou, Svensson, 2017, p. 13).

Later on Wright (2000) proposes a non-parametric alternative to the conventional VR test using ranges and signs that overcome biases and right-slanted sampling problems. Wright demonstrates that nonparametric variation sales, based on signs (S1 and S2) and ranks (R1 and R2), are better able to reject RWH violations than the tests suggested by Lo and Mackinlay.

Another test would be the Automatic Portmanteau Test for Serial Correlation. Based on the Box-Pierce $\mathrm{Q}$ test, Portmanteau test brings a less biased approach when testing linear dependency, in the sense that the researches does not have to specify the order of the autocorrelation tested, the asymptotic null distribution is chi-squared, with one 
degree of freedom, hence there is no need for bootstrapping. Also, the test is consistent and robust in presence of conditional heteroscedasticity of unknown form.

\section{Tests for nonlinear independence in the context of A.M.H.}

Due to the fact that the A.M.H. relies on the assumption that markets are characterized by cyclical efficiency, the testing of the hypothesis has to be empirically done also through statistical tests of nonlinear independence. In this context, one of the most important is represented by the BDS test.

Named after the initials of W. A. Brock, W. Dechert and J. Scheinkman, the BDS test is concerned with determining nonlinear dependence in time series. Even though it was not conceived as a leading indicator, it can, however aid in minimizing false detections of critical transitions caused by model misspecification. Fitting any linear model (e.g. $\operatorname{ARMA}(p, q), \operatorname{ARCH}(q)$ or GARCH(p,q) models) in order to remove linear structure from the time series after de-trending, the BDS determines validity of the hypothesized independence and identical distribution of residuals (null hypothesis).

Rejection of the hypothesis of independence and identical distribution may imply a hidden non-stationarity, a hidden nonlinearity or other type of structure not detected by de-trending and model fitting. The BDS test is bound to reject the independence and identical distribution hypothesis in the residual time series from a system approaching critical transition because these transitions are thought to be triggered by rather strong nonlinear responses. The utility of the BDS test lies in use as an ad-hoc diagnostic test concerning nonlinearities in time series before transitions - the early warning sign is highly unlikely to be a false positive as long as the BDS test rejects the independence and identical distribution hypothesis.

\section{Some important findings}

One of the first to test AMH is Kim et al. (2011), where in their study based on the DJIA Index, on a time frame of more than 100 years (1900-2009), they evaluate the adaptiveness of stock index using 3 methods: first they use the automatic VR and the non-parametric Portmanteau test in order to test for linear autocorrelation; secondly, they use the generalized spectral test for non-linear independence, and last they use dummy variables for different historical events in order to observe the relationship between return predictability and market conditions (Kim, Shamsuddin \& Lim, 2011, p.868-879). Their results suggest that the predictability of returns varies over time and that it is governed by changing market conditions.

Using a 5 year subsample of US (DJIA), UK (FT30) and Japan (TOPIX), Urquhart and Hudson (2013) tests the adaptiveness of market efficiency, and the time varying predictability of stock returns, using the following tests: the VR test for linear independence, McLeod Li test, Engle LM test, and BDS test (for nonlinear independence). According to their classification, a market is defined as adaptive when it has moved through three stages of dependence, and inefficient when there is no independence in returns throughout the sample (Soteriou, Svensson, 2017, p. 10).

In 2016 Urquhart and McGoarty (2016) study examines market conditions under times of bullish and bearish markets and normal returns, testing this way the AMH on S\&P500, 
FTSE100, NIKKEI225 and EURO STOXX 50 indices, in a timeframe between 1990 and 2014, using fixed-length moving subsamples. The study is important due to its contributions in understanding not only market conditions versus predictability patterns, but also the "behavior" of these conditions under different markets. Their findings are in line with the adaptive market hypothesis as return predictability changes over time for each market.

Andreas Soteriou and Louise Svensson (2017) examine the implications of the theoretical framework AMH on return predictability on the OMXS30 stock index between 1986 and 2014, using VR test for linear dependence, Chow and Denning joint test, the joint sign and rank test, and BDS test for non-linear independence. The results support the hypothesis of adaptiveness of stock return, as they fluctuate in close dependence to the market conditions.

Also, both Urquhart and McGoarty (2016) and Soteriou \& Svensson (2017) note that each market should be assessed individually due to the fact that different market behave differently in accordance with the AMH.(Soteriou, Svensson, 2017, p. 3).

\subsection{The Econophysics approach}

Another important emerging field is represented by the Econophysics, or "financial / statistical physics", a hybrid discipline situated at the boundaries of frames such as economics and physics.

Even though some roots of the field can be traced back at around 1970 when new developments in the domain of financial markets emerged, or within the works of Mandelbrot and fractal dimension, the notion was used for the first time by Stanley et al. (1996) during a conference on "Physics of Complex Systems", in 1995.

In the heterodox frame, both the A.M.H. and the Econophysics are trying to explain the complexity of financial markets from a "bottom up" perspective, hence "macroscopic" properties are viewed as the result of interactions at the level of the 'microscopic' constituents (Rickles, 2011, p.531-565). However, while the Adaptive Market Hypothesis tries to explain economic complexity based on an evolutionary perspective, Econophysics makes use of different models and concepts associated with the physics of complex systems in order to explain the various economical phenomena by providing physically realistic models and ultimately a theoretical basis.

Therefore, when speaking about Econophysics we can generally refer to a series of instruments, models and methods originally used and developed in the field of physics to the study of problems pertaining to the sphere of economics, more specifically finance.

Practically, from a financial standpoint, Econophysics aims to provide models and methods that replicate the statistical behaviours of stock prices and returns, and then apply these models for a better understanding of financial strategies and market mechanisms. Some fruitful innovations can be observed in the contributions of econophysicists to the modelling of out of equilibrium processes, signal detection in multivariate systems and information process and aggregation in multi-agents physical systems (Rickles, 2011, p.531-565). 
In this context, an important derivative of Econophysics is the statistical physics, a framework that allows "economic particles", namely investors, consumers, traders, to be rigorously analysed, as markets are perceived as complex macro-structures with an intricate internal system, in which the interactions of those "particles" generates the systemic properties. The adaptiveness of the system is explained by the fact that microstructural components are "reactive", and so the properties of the system are emergent, hence not reducible to some particular configuration of constituents' properties.

While the neo-classical finance theoristsmakes use of a series of traditional hypothesis such as (1) the absence of arbitrage opportunities; (2) rational agents, able to encompass a system which is heterogeneous by definition; (3)optimal processes of rational agents with respect to a given utility function; as starting points for building up new explicative models, the econophysicists assume that heterogeneous micro-interactions are too complex to be captured through the action of a representative agent (Schinckus, 2014, p.135-136). The premise accounts for the fact that the emergences of global behaviors are inferred, as the result of micro-units (investors, trader, consumers etc., as defined earlier) social interactions. This dynamic system is therefore characterized by a macro level in which the agents' heterogeneity is translated into a "collective activity", determined by the "indeterminism" existent at a micro level.

In physics, this is called the "multiple realizability argument”, and it may be a useful approach when trying to understand the "Stylized facts", respectively persistent macroregularities that cannot be described in terms of microeconomic theory, such as: the presence of heavy tails of financial distributions, volatility clustering, correlations, absence of autocorrelation in asset return dynamics, emergent phenomena in heterogeneous systems, etc. (Cont 2005, p.21.)

\section{Conclusions}

Based on the rational expectation principle, Efficient Market Hypothesis represents the cornerstone of modern finance. Main implications of the principle are related to the way investors are exploiting the market, in order to maximize their profits. While this arbitrage process, endogenous by definition, equalizes the market, as prices reflect all available information, the important question that arises is related to what causes the prices to change. For this concern EMH proposal lies in the statement „exogenous factors", and given their complexity and variety, are assumed to be modelled as random processes. However, as it can be observed in the real financial markets, prices do not behave in this „unbiased” way, and so the emergence of new models and frameworks in order to explain the various economical phenomena is needful.

Two of these new approaches are The Adaptive Market Hypothesis, and the field of Econophysics.

The hypothesis of adaptable markets proposed by Andrew Lo tries to explain economic complexity based on an evolutionary perspective, and even thou it is still in an early stage of development, seems to represent the mechanisms of the financial markets better, taking into consideration a larger spectrum of variables. In the post-modern world, the A.M.H.'s feasibility rises along with the capacity of the socio-economic 


\section{-}

environment to be influenced by the rapid expansion of technology and implicitly by the capacity to collect massive amounts of information pertaining to financial markets mechanisms and dynamics and investor behavior.

On the other hand, Econophysics makes use of different models and concepts associated with the physics of complex systems in order to explain the various economical phenomena by providing physically realistic models and ultimately a theoretical basis. Econophysicists assume that heterogeneous micro-interactions are too complex to be captured through the action of a representative agent (Schinckus, 2014, p.135-136) as they are both subject and determinant to system changes, resulting in a process in which a micro-indeterminism is inducing macro-determinism.

Even thou between the two theories exists differences such as explanatory perspective: evolutionary (AMH) vs. physical laws (Econophysics), views over the economic system: adaptive vs. self-organizing adaptive system, stage of empirical development etc., they both deserve a central role in further discussions in the journey of understanding the complex mechanisms of financial markets.

\section{References}

Butler, M. \& Kazakov, D., 2012. Testing implications of the Adaptive Market Hypothesis via computational intelligence. In 2012 IEEE Conference on Computational Intelligence for Financial Engineering and Economics, CIFEr 2012 - Proceedings. pp. 249-256.

Cont, R., 2005. Volatility Clustering in Financial Markets: Empirical Facts and Agent Based Models. A Kirman \& G Teyssiere (eds.): Long memory in economics, p.21.

Fama, E. F. (1965). The Behavior of Stock-Market Prices. The Journal of Business, 38(1), p.34.

Fama, E. F., (1991). Efficient Capital Markets: II. The Journal of Finance, 46(5), p.1575.

Hoque, H.A.A.B., Kim, J.H. \& Pyun, C.S., 2007. A comparison of variance ratio tests of random walk: A case of Asian emerging stock markets. International Review of Economics and Finance, 16(4), pp.488-502.

Kim, J.H., 2006. Wild bootstrapping variance ratio tests. Economics Letters, 92(1), pp.38-43.

Kim, J.H., Shamsuddin, A. \& Lim, K.P., 2011. Stock return predictability and the adaptive markets hypothesis: Evidence from century-long U.S. data. Journal of Empirical Finance, 18(5), pp.868-879.

Lo, A. and MacKinlay, A. (1988). Stock Market Prices Do Not Follow Random Walks: Evidence from a Simple Specification Test. Review of Financial Studies, 1(1), pp.4166.

Lo, A. (2004). The Adaptive Markets Hypothesis. The Journal of Portfolio Management, 30(5), pp.15-29.

Rickles, D., 2011. Econophysics and the Complexity of Financial Markets. In Philosophy of Complex Systems. pp. 531-565.

Soteriou, A., Svensson, L., 2017. "Testing the Adaptive Market Hypothesis on the OMXS30 Stock Index: 1986-2014”, Master thesis, Jönköping University, Jönköping, Sweden. 
Schinckus, C., 2014. Stylized facts and multiple realizability in Econophysics. Results in Physics, 4, pp.135-136. 